\title{
Recent CMS results on soft and small-x QCD physics
}

\author{
Rajat Gupta $^{1}$ on behalf of the CMS Collaboration \\ ${ }^{1}$ Panjab University, Chandigarh, India
}

\begin{abstract}
We present latest results of soft and small-x QCD measurements with the CMS experiment, such as minimum bias/underlying event physics, and studies on forward jet production.
\end{abstract}

\subsection{Introduction}

Quantum chromodynamics (QCD), the theory of strong nuclear interactions, is undoubtedly a very rich and successful theory. There are interesting phenomena in nuclear interactions which have yet to be seen, phenomena that are ultimately related to the properties of color confinement and asymptotic freedom of the strong interactions. These effects can take place in special corners of phase space accessible at the Large Hadron Collider (LHC). More concretely, it is not clear whether a gluon-gluon recombination mechanism takes place at low values of the fraction of the nucleon momentum x carried by its partonic constituents. Said mechanism is believed to slow down the rapid growth of the nucleon's structure function at very small values of $\mathrm{x}$.

On the other hand, we have to refine our understanding of the underlying dynamics in low momentum exchange processes in hadronic collisions. The description of these effects rely on phenomenological models, whose parameters are tuned based on fits to data. Dedicated measurements provide valuable inputs for Monte Carlo event generators, which are of great importance for precision measurements of Standard Model processes and searches for New Physics at the LHC. [1]:

In this context, we briefly discuss the following recent results by the CMS experiment

- Measurement of the inelastic proton-proton cross section at $\sqrt{s}_{\mathrm{s}}=13 \mathrm{TeV}$ [2];

- Measurement of charged particle spectra in minimum-bias events from protonproton collisions at $\sqrt{\mathrm{s}}_{\mathrm{s}}=13 \mathrm{TeV}[3]$;

- Femtoscopic Bose-Einstein Correlations of charged hadrons in proton-proton collisions at $\sqrt{\mathrm{s}}_{\mathrm{s}}=13 \mathrm{TeV}[4]$;

- Measurement of light-by-light scattering in ultraperipheral $\mathrm{PbPb}$ collisions at $\bigvee_{\mathbf{s N N}}=5.02 \mathrm{TeV}[5]$;

- Exclusive $\rho(770)^{\circ}$ photoproduction in ultra-peripheral $\mathrm{pPb}$ collisions at $\sqrt{\mathrm{sNN}}_{\mathrm{S}}=5.02 \mathrm{TeV}$ with the CMS experiment [6];

- Study of the underlying event in top quark pair production at $13 \mathrm{TeV}$ [7]; 
- Measurement of the underlying event activity in inclusive $\mathrm{Z}$ boson production in proton-proton collisions at at $\sqrt{\mathrm{s}}=13 \mathrm{TeV}$ [8];

- Constraints on the double-parton scattering cross section from same-sign W boson pair production in proton-proton collisions at $\sqrt{s}_{\mathbf{s}}=8 \mathrm{TeV}$ [9];

- Measurement of double parton scattering in same-sign WW production in p-p collisions at $\sqrt{\mathrm{s}}_{\mathrm{s}}=13 \mathrm{TeV}$ with the CMS experiment [10].

\subsection{Measurement of the inelastic proton-proton cross section at $\sqrt{s}=13 \mathrm{TeV}$}

The total proton-proton (p-p) cross section can be identified as the sum of two terms: the elastic scattering contribution and the inelastic scattering contribution. The latter can be further factorised into four independent processes: the single diffractive (SD), the double diffractive (DD), the central diffractive (CD) and the non-diffractive (ND) components. At the LHC energy scale, about $20 \%$ of the scattering processes count as elastic and the remaining $80 \%$ can be addressed as inelastic interactions.

The CMS experiment measured the inelastic p-p cross section at a centre of mass energy of $\sqrt{s}_{\mathrm{s}}=13 \mathrm{TeV}$ based on events with energy deposits in the forward calorimeter, which cover pseudorapidities of $-6.6<\eta<-3.0$ and $3<\eta<5.2$. Defining $\mathrm{M}_{\mathrm{x}}$ and $\mathrm{M}_{\mathrm{y}}$ as the masses of the diffractive dissociation systems at negative and positive pseudorapidities respectively, the fiducial cross section is measured in a phase space region corresponding to $M_{x}<4.1 \mathrm{GeV}$ and $\mathrm{M}_{\mathrm{y}}<13 \mathrm{GeV}$. In other terms $\xi_{x}=\frac{M_{x}^{2}}{s}$ and $\xi_{y}=\frac{M_{y}^{2}}{s}$ and thus $\xi=\max \left(\xi_{x}, \xi_{y}\right)$. The cross section is then calculated as $\sigma=\frac{N_{\text {int }}\left(1-b_{\xi}\right)}{\epsilon_{\xi^{L}}}$ where $\mathrm{N}_{\text {int }}$ is the number of interactions, $\epsilon_{\xi}$ is the efficiency defined as the fraction of selected stable-particle events that fulfill the detectorlevel offline selection criteria, and the contamination $b_{\xi}$ is the fraction of events selected at detector level that are not part of the considered stable-particle phase space domain. The CMS experimental results are reported in Fig. 1 for two different phase space domains, compared to ATLAS results and many Monte Carlo predictions.

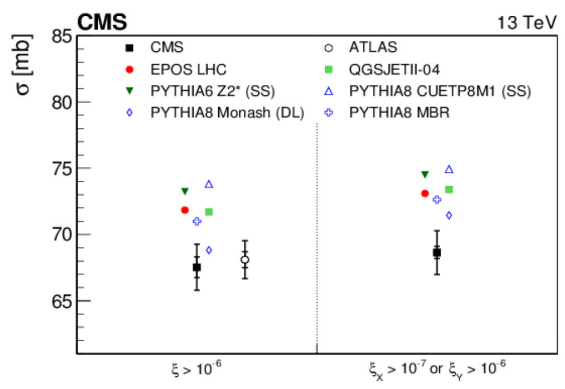

Fig. 1. Proton-proton inelastic cross section at $\sqrt{s}_{s}=13 \mathrm{TeV}$ in two phase space regions, where $\xi=M^{2} / s$, compared to different models and to the ATLAS result [2].

An inelastic cross section of $67.5 \pm 0.8$ (syst) \pm 1.6 (lumi) $\mathrm{mb}$ is obtained for $\xi=M^{2} / s>10^{-6}$ (corresponding to $\mathrm{M}>13 \mathrm{GeV}$ ), with $\mathrm{M}$ the larger of $\mathrm{M}_{\mathrm{x}}$ and $\mathrm{M}_{\mathrm{y}}$. This result is consistent with a previous measurement in the same phase space. In addition, an inelastic cross section of $68.6 \pm 0.5$ (syst) \pm 1.6 (lumi) $\mathrm{mb}$ is obtained in the enlarged phase space $\xi_{\mathrm{x}}$ $>10^{-7}$ and/or $\xi>10^{-6}$ (corresponding to $\mathrm{M}_{\mathrm{x}}>4.1 \mathrm{GeV}$ and/or $\mathrm{M}_{\mathrm{y}}>13 \mathrm{GeV}$ ). The measured cross sections are smaller than those predicted by the majority of models for hadron-hadron scattering, as previously observed in pp collisions at $\sqrt{s}_{\mathrm{s}}=7 \mathrm{TeV}$. Given that the difference 
between the two sets of measurements is dominated by the contribution from low-mass diffractive processes, the data-model discrepancies observed here suggest a theoretical underestimation of the cross section for such events.

\subsection{Measurement of charged particle spectra in minimum-bias events from proton-proton collisions at $\sqrt{ } \mathrm{s}=13 \mathrm{TeV}$}

Particle production without any selection bias arising from the requirement of the presence of a hard scattering process is known as minimum bias (MB). The bulk of these events occur at low momentum exchanges between the interacting partons inside the hadrons, where diffractive scattering or multiple partonic interactions (MPI) play a significant role.

One can characterize MB events by means of charged particle distributions. Charged particle distributions are measured for charged particles with $\mathrm{p}_{\mathrm{T}}>0.5 \mathrm{GeV}$ and $|\eta|<2.4$ for events collected with the CMS MB trigger. The measured distributions are presented for different event data samples based on the calorimeter activity in the forward region by requiring the presence of at least one tower with energy above $5 \mathrm{GeV}$ in the acceptance region $3<|\eta|<5$, and in some cases with a veto condition for towers less than a given threshold value. The different event classes are as non-single diffractive enriched sample (NSDenhanced) when there is calorimeter activity in both sides, as single diffractive enriched (SDenhanced) when there is calorimeter activity on one side and a veto on the opposite side, and as inelastic when there is calorimeter activity on at least one side of CMS. The distribution labelled as SD-One-Side enhanced sample corresponds to the symmetrized distribution constructed from the SD-minus and SD-plus enhanced samples.

The normalized particle distribution is measured as a function of the charged particle pseudorapidity for the three different selections (see Fig. 2). The results are unfolded to particle level. PYTHIA8 CUETM1, PYTHIA8 MBR 4C and EPOS LHC results are compared to the data. PYTHIA8 MBR 4C describes reasonably well the data for the SDenhanced samples, but overestimates the yield in central pseudorapidities for the nondiffractive samples. PYTHIA8 CUETM1 and EPOS LHC give a fair description for the nondiffractive samples, but they are off w.r.t. data for the SD-enhanced selection.

\subsection{Femtoscopic Bose-Einstein Correlations of charged hadrons in proton- proton collisions at $\sqrt{ }=13 \mathrm{TeV}$}

Femtoscopic Bose-Einstein correlations (BEC) can be used to probe the size and shape of the particle emitting region in high energy collisions. These femtoscopic techniques are employed to characterize the emission region at the freeze-out stage of the evolving system. Three analysis methods, each with a different dependence on MC simulations, were used to generate correlation functions. These three methods are Double Ratio (DR) technique, Cluster subtraction (CS) technique and Hybrid Cluster Subtraction (HCS) technique. The value of length of homogeneity $\mathrm{R}_{\mathrm{inv}}$ and correlation intensity $\lambda$ obtained with each of three methods as a function of $\left\langle N_{\text {tracks }}\right\rangle$ (average multiplicity at particle level, i.e. corresponding to the measured $N_{\text {track }}^{\text {offline }}$ corrected for acceptance and efficiency) and $k_{T}$ are shown in Fig. 3. The radius fit parameter increases as a function of the multiplicity, showing a change in slope around $\left\langle N_{\text {tracks }}\right\rangle \sim 20-30$ and a tendency to saturate at higher multiplicities. For the DR and HCS procedures, the intercept parameter rapidly decreases for increasing multiplicities in the very small $\left\langle N_{\text {tracks }}>\right.$ region, above which it shows an almost constant 

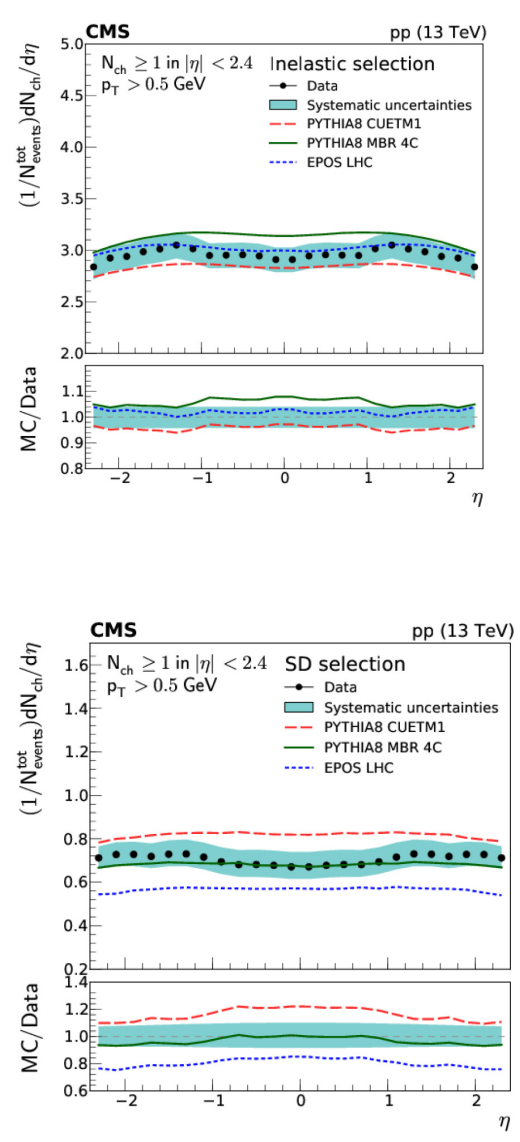
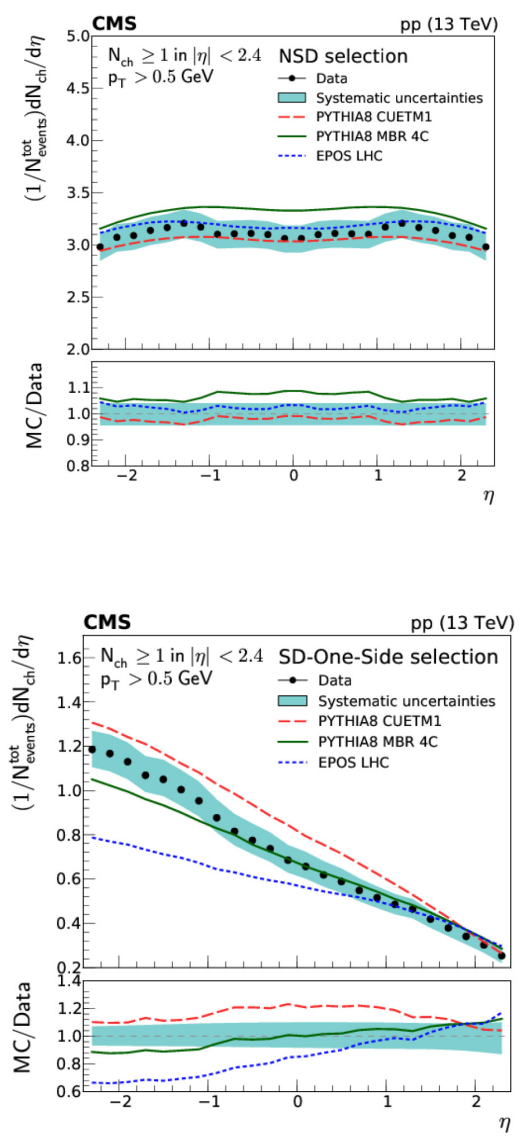

Fig. 2. Normalized differential charged particle multiplicity distributions as a function of pseudorapidity for the different sample selections. The error band represents the total systematic uncertainty [3].

value with increasing $\left\langle N_{\text {tracks }}\right\rangle$. The lengths of homogeneity tend to decrease when increasing $k_{T}$, more so at lower multiplicity. This behaviour is compatible with an emitting source that was expanding prior to decoupling. The correlation intensity $\lambda$ also decreases with increasing values of $k_{T}$, with a more pronounced slope than that for $R_{i n v}$.

Finally, in hydrodynamic models, valuable information about the collective transverse expansion of the system (transverse flow) can be obtained from the slope of a linear fit to $1 / R_{i n v}^{2}$ versus the transverse mass, $m_{T}=\sqrt{m_{\pi}^{2}+<k_{T}>^{2}}$ (where $m_{\pi}$ is the pion mass). In addition, the value of $1 / R_{i n v}^{2}$ at $m_{T}=0$ reflects the final-state geometrical size of the source. Fig. 4 shows $1 / R_{\text {inv }}^{2}$ versus $m_{T}$ for a variety of multiplicity ranges. The left plot shows that the expansion in the low multiplicity region is faster than in the high multiplicity region. The corresponding geometrical sizes are $R_{G}^{M B}=5.1 \pm 0.4$ (stat.) fm and $R_{G}^{H M}=4,2 \pm$ 1.1 (stat.) fm, for the low and high multiplicity regions, respectively. The right plot shows the variation of $1 / R_{i n v}^{2}$ with $m_{T}$ in finer multiplicity ranges, showing in more detail the evolution of the slope: the collective flow decreases with increasing multiplicity, but this trend seems to saturate around a reconstructed multiplicity of $\sim 80$. Therefore, the present analysis reveals additional similarities of the systems produced in high multiplicity pp collisions and those found using data for larger initial systems. These results may provide 
additional constrains for future attempts using hydrodynamical models and/or the CGC framework to explain the entire range of similarities between high multiplicity pp and heavy ion interactions.
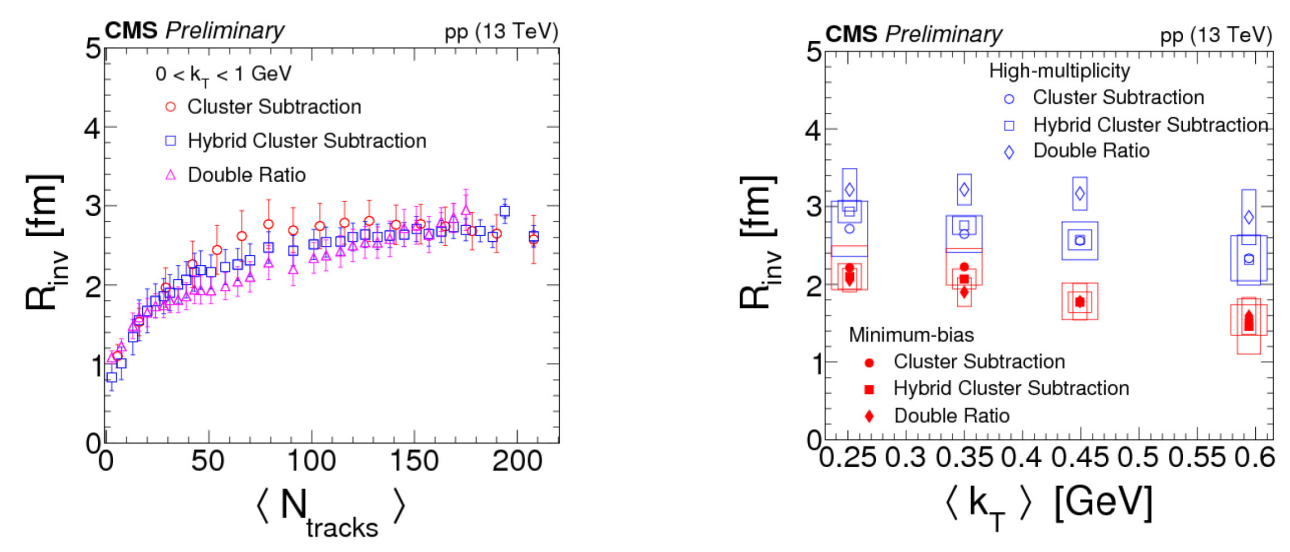

Fig. 3. Results for $R_{i n v}$ as a function of multiplicity and $k_{T}$. In the left plot, statistical and systematical uncertainties are represented by internal and external error bars, respectively. In the right plot, statistical and systematic uncertainties are shown as error bars and open boxes, respectively [4].
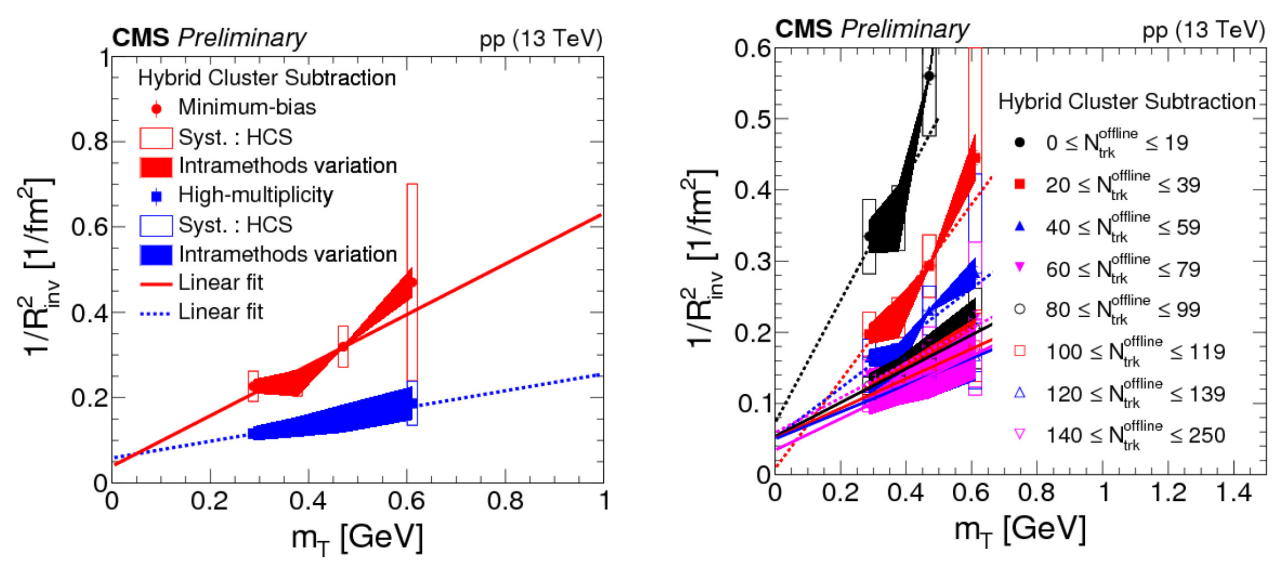

Fig. 4. $1 / R_{\text {inv }}^{2}$ as a function of $m_{T}=\sqrt{\left.m_{\pi}^{2}+<k_{T}\right\rangle^{2}}$ for the HCS method. The MB range corresponds to $0 \leq N_{\text {trk }}^{\text {offline }} \leq 79$ and the $\mathrm{HM}$ one, to $80 \leq N_{\text {trk }}^{\text {offline }} \leq 250$. Statistical uncertainties are represented by error bars, systematic uncertainties related to the HCS method are shown as open boxes and the relative uncertainties from the intramethods variation are represented by the shaded bands. Only statistical uncertainties are considered in all the fits [4].

\subsection{Measurement of light-by-light scattering in ultraperipheral $\mathrm{PbPb}$ collisions at $\sqrt{ } \mathrm{NNN}=5.02 \mathrm{TeV}$}

A measurement of light-by-light scattering (LbL), $\gamma \gamma \rightarrow \gamma \gamma$, in ultraperipeheral $\mathrm{PbPb}$ collisions at $\sqrt{S}=5.02 \mathrm{TeV}$. Diphotons are required to have transverse energy $E_{T}^{\gamma}>2 \mathrm{GeV}$, pseudorapidity $\left|\eta_{\gamma}\right|<2.4$, invariant mass $m^{\gamma \gamma}>5 \mathrm{GeV}$, transverse momentum $p_{T}^{\gamma \gamma}<1$ 
$\mathrm{GeV}$, and acoplanarity $A_{\phi}=\left(1-\frac{\Delta \phi^{\gamma \gamma}}{\pi}\right)<0.01$. Only $14 \mathrm{LbL}$ events are observed after all selection criteria applied. MADGRAPH v5 Monte Carlo (MC) event generator including the modifications given in $[11,12]$ is used to generate the LbL signal. Fig. 5 shows the comparison of the simulated and measured distributions of diphoton invariant mass $m^{\gamma \gamma}$, diphoton transverse momentum $p_{T}$, and the diphoton acoplanarity $A_{\phi}$. Measured yields as well as kinematic distributions are found to be in good agreement with the combination of the LbL scattering signal plus QED $e^{+} e^{-}$and CEP+other background expectations. Evidence of LbL scattering is observed with a signal significance of 4.1 standard deviation (4.4 standard deviations expected). Cross section of the measured fiducial LbL scattering,

$$
\sigma_{\text {fid }}(\gamma \gamma \rightarrow \gamma \gamma)=120 \pm 46(\text { stat }) \pm 28 \text { (syst) } \pm 4 \text { (theo) } \mathrm{nb}
$$

is found to be consistent with the standard model prediction.
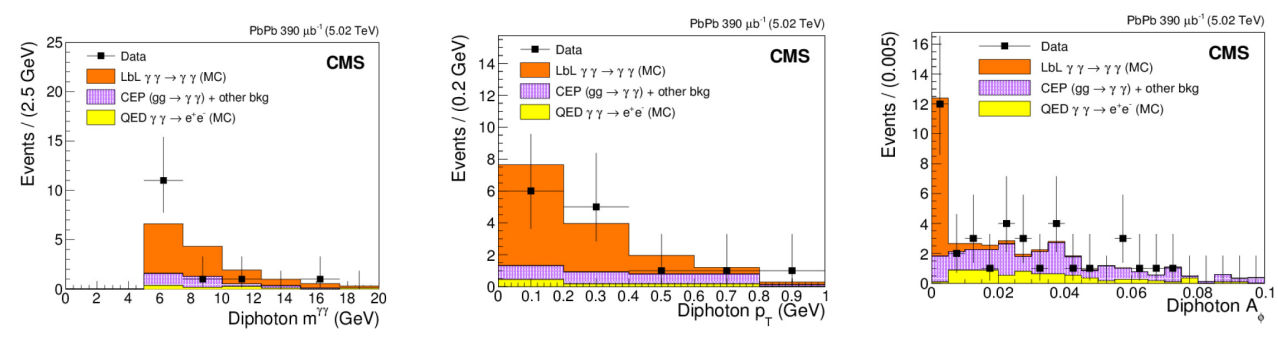

Fig. 5. Distributions of diphoton invariant mass $m^{\gamma \gamma}$ (left), transverse momentum $p_{T}$ (middle), and acoplanarity $A_{\phi}$ (right) [5].

\subsection{Exclusive $\rho(770)^{0}$ photoproduction in ultra-peripheral $\mathrm{pPb}$ collisions at $\sqrt{ } \mathrm{sNN}=5.02 \mathrm{TeV}$ with the CMS experiment}

A first measurement of exclusive $\rho(770)^{\circ}$ photoproduction, $\gamma p \rightarrow \rho(770)^{\circ} p$, in ultraperipheral $\mathrm{pPb}$ collisions at $\sqrt{S_{N N}}=5.02 \mathrm{TeV}$ has been performed with the CMS experiment. In this analysis, the cross section of exclusive photoproduction of $\rho(770)^{\circ}$ meson in the $\pi^{+} \pi^{-}$decay channel is measured as a function of the photon-photon centre-of-mass energy $\left(W_{\gamma p}\right)$ and the squared four-momentum transfer at the proton vertex $\left(|t| \approx p_{T}^{2}\right)$. In order to simulate exclusive $\rho(770)^{o} \rightarrow \pi^{+} \pi^{-}$photoproduction decay, the STARLIGHT (version 2.2.0) $\mathrm{MC}$ event generator is utilized. Exclusive $\rho(770)^{\circ}$ photoproduction cross section as a function of $W_{\gamma p}$ and $|t|$ in the rapidity interval $1.2<y_{\pi^{+} \pi^{-}}<0$ is shown in Fig. 6 . The results are compared to fixed target and HERA (H1 and ZEUS) experiments. Both CMS data and the earlier ones show a good agreement with the theoretically inspired fits.

\subsection{Underlying Event Measurements}

The underlying events (UE) surround the hard scattering of a hadronic interaction and can receive contributions from initial and final state radiation (ISR/FSR), QCD evolution or CR and additional partonic interactions in the same collision. Measuring the UE is crucial for a proper understanding of the interaction and for $\mathrm{MC}$ model tuning. 

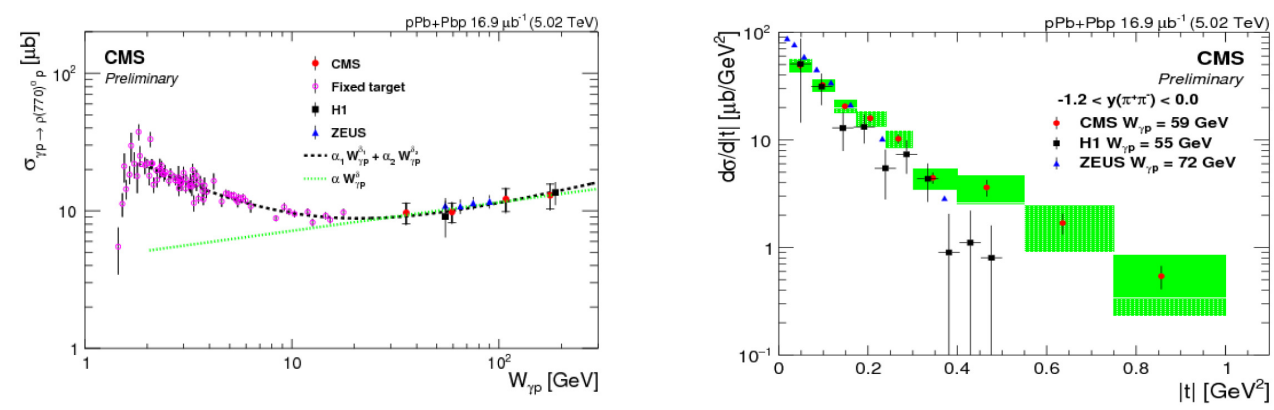

Fig. 5. Differential cross section for exclusive $\rho(770)^{\circ}$ photoproduction as a function of $W_{\gamma p}$ (left) and $|t|$ in the rapidity interval $1.2<y_{\pi^{+} \pi^{-}}<0$ (right) [6].

Fig. 6 shows the azimuthal plane region segmentations once the leading object is identified and defining $|\Delta \phi|$ as the relative azimuthal distance between a charged particle and the leading object:

- Towards: $|\Delta \phi|<60^{\circ}$

- Transverse: $60^{\circ}<|\Delta \phi|<120^{\circ}$

- Away: $|\Delta \phi|>120^{\circ}$

Two kinds of analyses done within the CMS Collaboration are presented and the main difference between the two is the choice of the leading object.

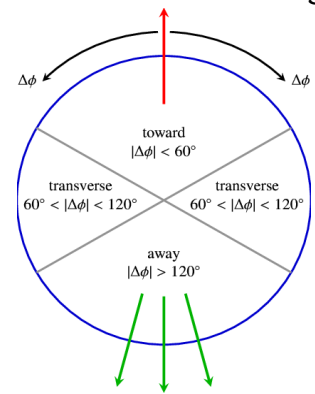

Fig. 6. The azimuthal plane sections in the UE studies. The different regions are identified starting from the identification of the leading object of the event.

\subsubsection{Study of the underlying event in top quark pair production at $13 \mathrm{TeV}$}

The study of the UE in $t \bar{t}$ events provides a direct test of its universality at higher energy scales than those probed in minimum bias or DY events. This is relevant as a direct probe of color reconnection (CR), which is needed to confine the initial QCD color charge of the $t$ quark into color-neutral states. The analysis is performed using final states where both of the W bosons decay to leptons, yielding one electron and one muon with opposite charge sign, and the corresponding neutrinos. In addition, two $b$ jets are required in the selection, as expected from the $t \bar{t} \rightarrow(e v b)(\mu v b)$ decay. This final state is chosen because of its expected high purity and because the products of the hard process can be distinguished with high 
efficiency and small contamination from objects not associated with t quark decays, e.g., jets from ISR.

Various characteristics, such as the multiplicity of the selected charged particles, the flux of momentum, and the topology or shape of the event have different sensitivity to the modeling of the recoil, the contribution from MPI and CR, and other parameters, and hence used to characterize UE activity. Fig. 7 (left) shows the normalized differential cross section measured as function of $\mathrm{N}_{\mathrm{ch}}$. The majority of the distributions analyzed indicate a fair agreement between the data and the POWHEG + PYTHIA8 setup with the CUETP8M2T4 tune, but disfavour the setups in which MPI and CR are switched off, or in which $\alpha_{S}^{F S R}\left(M_{Z}\right)$ is increased. The data also disfavour the default configurations in HERWIG++, HERWIG7, and SHERPA. It has been furthermore verified that, as expected, the choice of the next-toleading order matrix-element generator does not impact significantly the expected characteristics of the UE by comparing predictions from POWHEG and MADGRAPH5_aMC@NLO, both interfaced with PYTHIA8. The sensitivity of these results to the choice of $\bar{\alpha}_{S}^{F S R}\left(M_{Z}\right)$ in the parton shower is tested by performing a scan of the $\chi^{2}$ value as a function of $\alpha_{s}^{I S R}\left(M_{Z}\right)$ or $\alpha_{S}^{F S R}\left(M_{Z}\right)$ as shown in Fig. 7 (right). A value of $\alpha_{s}^{F S R}\left(M_{Z}\right)=$ $0.120 \pm 0.006$ is obtained, which is lower than the one assumed in the Monash tune and used in the CUETP8M2T4 tune. The new CMS default tune CP5 uses $\alpha_{S}^{F S R}\left(M_{Z}\right)=\alpha_{S}^{I S R}\left(M_{Z}\right)$ $=0.118[13]$.
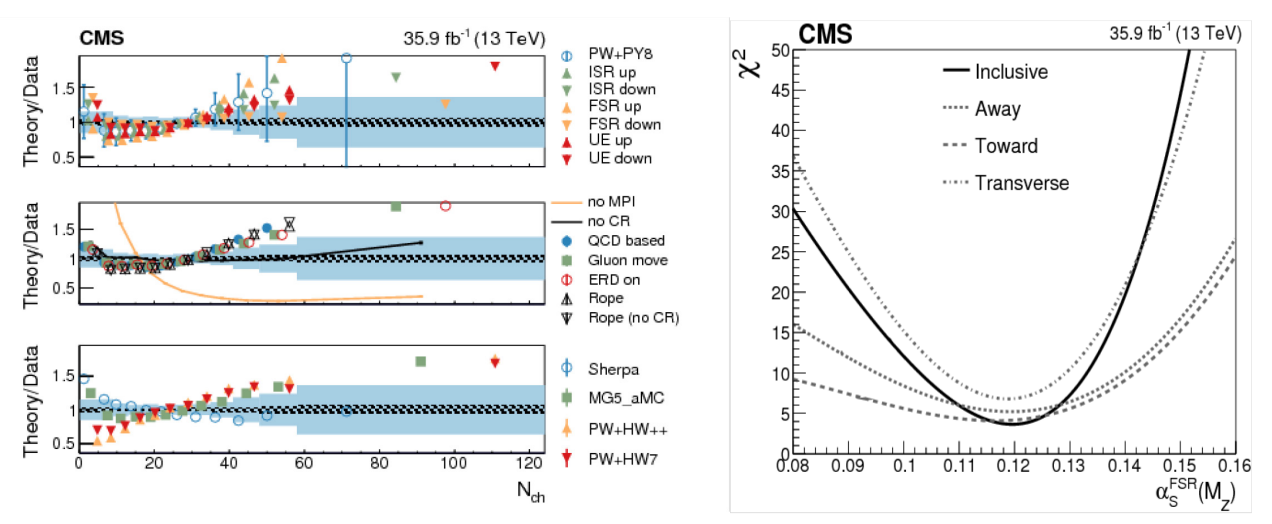

Fig. 7. The normalized differential cross section as a function of $\mathrm{N}_{\mathrm{ch}}$ compared to predictions of different models (left), scan of the $\chi^{2}$ as a function of the value of $\alpha_{S}^{F S R}\left(M_{Z}\right)$ employed in PW+P8 simulation [7].

\subsubsection{Measurement of the underlying event activity in inclusive $Z$ boson production in proton-proton collisions at at $\sqrt{ } s=13 \mathrm{TeV}$}

A measurement of UE activity using events with inclusive $Z \rightarrow \mu^{+} \mu^{-}$production is performed in pp collisions at the centre-of-mass energy of $\sqrt{s}=13 \mathrm{TeV}$. Since production of $\mathrm{Z}$ boson process experimentally provides a clean signal, such a process can be utilized for clear identification of the UE activity. The observables, the total number of charged particles (particle density) and the scalar sum of their $p_{T}\left(\Sigma p_{T}\right.$ density), are used to quantify the UE activity. In the analysis, all selected $\mathrm{Z}$ boson events are required to have the tracks with $p_{T}>$ $0.5 \mathrm{GeV}$ and $|\eta|<2$. The results are corrected to the stable charged particle level by using iterative D'Agostini method. In order to understand the MPI evolution with centre-of-mass energies, measurements are also compared with previous results from Tevatron and LHC. Fig. 8 (left) shows the UE activity as a function of $p_{T}^{\mu \mu}$, compared with different $\mathrm{MC}$ 
simulation in transverse region. POWHEG + HERWIG++ with tune EE5C overestimates UE activity by $10-15 \%$ in all regions, POWHEG + PYTHIA8 with CUETP8M1 tune describes the data within 5\%, whereas MADGRAPH + PYTHIA8 with CUETP8M1 tune gives the best description. Fig. 8 (right) shows the UE activity as a function of $p_{T}^{\mu \mu}$ at the centre-of-mass energy of 1.96, 7 and $13 \mathrm{TeV}$ in transverse region. POWHEG + HERWIG++ overestimates data by $40 \%$ at $1.96 \mathrm{TeV}$ and upto $10 \%$ at $13 \mathrm{TeV}$, whereas POWHEG + PYTHIA8 describes data within $10 \%$ at $1.96 \mathrm{TeV}$ to $5 \%$ at $13 \mathrm{TeV}$.
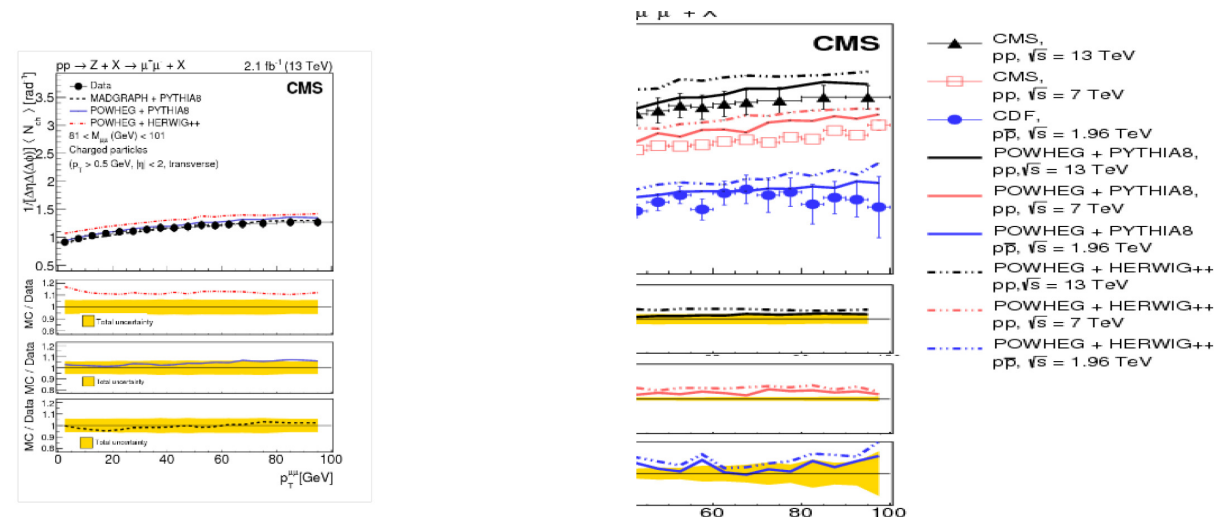

Fig. 7. Comparison of UE activity measured at $\sqrt{s}=13 \mathrm{TeV}$ compared with several MC predictions (left) and Comparison of UE activity measured at $\sqrt{s}=13 \mathrm{TeV}$ with the measurements at $7 \mathrm{TeV}$ [14] and $1.96 \mathrm{TeV}$ [15], by the CMS and CDF experiments for particle density in the transverse region as a function of $p_{T}^{\mu \mu}[8]$.

\subsection{Double Parton Scattering Measurements}

The study of DPS processes provides valuable information on the transverse distribution of partons in the proton and on the parton correlations in the hadronic wave function. Study of the same-sign WW production via DPS, from p-p collisions at $\sqrt{s}=8$ and $13 \mathrm{TeV}$ at integrated luminosity of $19.7 \mathrm{fb}^{-1}$ and $35.9 \mathrm{fb}^{-1}$ respectively. The study is performed using same-sign $\mathrm{W}$ boson pair final state, with each boson decaying leptonically. In order to enhance the sensitivity, the $\mu^{ \pm} \mu^{ \pm}$final state is combined with the $e^{ \pm} \mu^{ \pm}$channel. A multivariate analysis has been performed in order to enhance the signal sensitivity; a limit on the DPS yield, along with corresponding $\sigma_{e f f}$, has been estimated. Figure 8 shows the distribution of the BDT classifier in $\mu^{ \pm} \mu^{ \pm}$channel at 8 (left) and $13 \mathrm{TeV}$ (middle) respectively. At $8 \mathrm{TeV}$, although no direct measurements of DPS yield are made with current statistics, exclusion limit on DPS signal strength was set. BDT response shape gives the limit estimation, excluding at 95\% CLs a signal strength $r>1.897$ (28 DPS events), with an expected exclusion of $r>2.01$ (30 DPS events), which means an upper limit on $\sigma_{W W}^{D P S}<1.12$ $\mathrm{pb}$ at $95 \%$ of confidence level. Considering the two scattering to be independent and no correlation between interacting partons, the lower limit on $\sigma_{\text {eff }}$ can be written as, $\sigma_{\text {eff }}>$ $5.91 \mathrm{mb}$. At $13 \mathrm{TeV}$, the expected cross section and significance from PYTHIA8 was 1.64 $\mathrm{pb}$ and $3.27 \sigma$ respectively, but the measured cross section is below the expected value at $1.09_{-0.49}^{+0.50} \mathrm{pb}$ with a significance of $2.23 \sigma$. The upper limit on the cross section in the absence of signal is expected to be $<0.97 \mathrm{pb}$ and measured to be $<1.94 \mathrm{pb}$. The measurements of 
effective cross section including final stated with photons, vector bososns and jets are summarized in Fig. 8 (right).
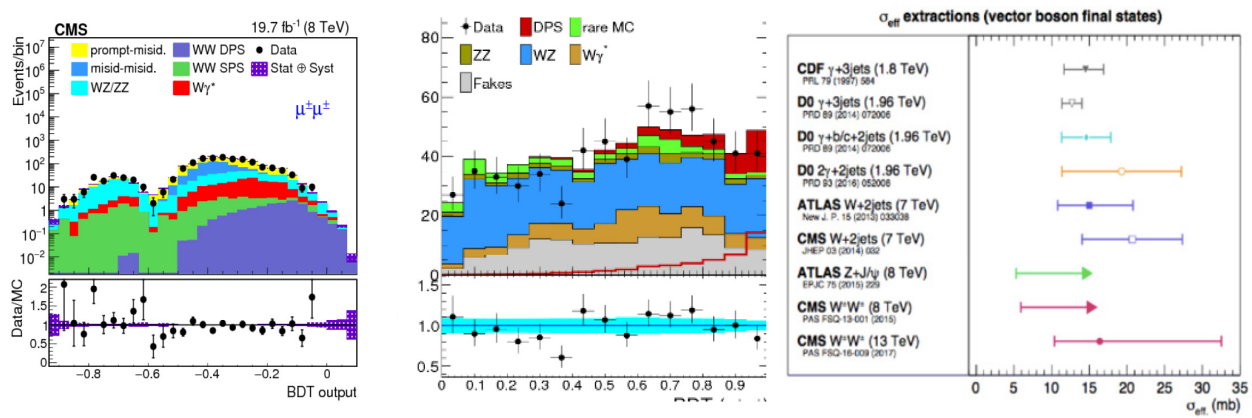

Fig. 7. Final BDT classifier output with all background estimations in place for $\mu^{ \pm} \mu^{ \pm}$channel at 8 (left) [9] and $13 \mathrm{TeV}$ (middle) [10], Right: comparison of extracted $\sigma_{\text {eff }}$ using various processes at the previous experiments and CMS measurements [10].

\section{References}

1. CMS Collaboration, JINST 3 S8004 (2008)

2. CMS Collaboration, JHEP 07 (2018) 161, doi: 10.1007/JHEP07(2018)161

3. CMS Collaboration, Eur. Phys. J. C (2018) 78: 697, doi: 10.1140/epjc/s10052-0186144-y

4. CMS Collaboration, CMS-PAS-FSQ-15-009 (2018). http://cds.cern.ch/record/2318575

5. CMS Collaboration, CMS-FSQ-16-012, CERN-EP-2018-271, Submitted to Phys. Lett. B. (2018). [arXiv:1810.04602]

6. CMS Collaboration, CMS-PAS-FSQ-16-007 (2018). http://cds.cern.ch/record/2636688

7. CMS Collaboration, CMS-TOP-17-015, CERN-EP-2018-271, Submitted to EPJC. (2018). [arXiv:1807.02810v1]

8. CMS Collaboration, JHEP 07 (2018) 032, doi: 10.1007/JHEP07(2018)032

9. CMS Collaboration, JHEP 02 (2018) 032, doi: 10.1007/JHEP02(2018)032

10. CMS Collaboration, CMS-PAS-FSQ-16-009 (2018). https://cds.cern.ch/record/2257583

11. D. d'Enterria and G. G. da Silveira, Phys. Rev. Lett. 111 (2013) 080405, doi: 10.1103/PhysRevLett.111.080405 [Erratum: doi: 10.1103/PhysRevLett.116.129901]

12. D. d'Enterria and J.-P. Lansberg, Phys. Rev. D 81 (2010) 014004, doi: 10.1103/PhysRevD.81.014004

13. CMS Collaboration, CMS-PAS-GEN-17-001 (2018). http://cds.cern.ch/record/2636284

14. CMS Collaboration, Eur. Phys. J. C (2012) 72: 2080, doi: 10.1140/epjc/s10052-0122080-4

15. CMS Collaboration, Phys. Rev. D 82 (2010) 034001, doi: 10.1103/PhysRevD.82.034001 